\title{
Delegation and Public Pressure in a Threshold Public Goods Game
}

\begin{abstract}
Many public goods cannot be provided directly by interested parties (e.g. citizens), as they entail decision-making at nested hierarchical scales: at a lower level individuals elect a representative, while at a higher scale elected delegates decide on the provision level, with some degree of scrutiny from their constituency. Furthermore, many such decisions involve uncertainty about the magnitude of the contribution that is needed for the good to be provided (or bad to be avoided). In such circumstances delegates can serve as important vehicles for coordination by aggregating societal preferences for provision. Yet, the role of delegation in threshold public goods games is understudied. We contrast the behavior of delegates to that of self-representing individuals in the avoidance of a public bad in an experimental setting. We randomly assign twelve subjects into four teams and ask each team to elect a delegate via majority voting. The elected delegates play several variants of a one-shot public goods game in which losses can ensue if the sum of their contributions falls short of a threshold. We find that when delegation is coupled with a mild form of public pressure, it has a significantly negative effect on contributions, even though the non-delegates can only signal their preferred levels of public good contributions. The reason is that delegates give more weight to the least cooperative suggestion: they focus on the lower of the two public good contributions recommended by their teammates.
\end{abstract}

Keywords: delegation; cooperation; threshold public goods game; climate experiment.

JEL codes: C72; C92; D81; H4; Q54

Doruk İriş: Sogang University, Department of Economics. dorukiris@sogang.ac.kr

Jungmin Lee: Seoul National University and Institute for the Study of Labor (IZA). imlee90@snu.ac.kr

Alessandro Tavoni: Department of Economics, University of Bologna and London School of Economics, Grantham Research Institute. https://orcid.org/0000-0002-2057-5720 a.tavoni@Ise.ac.uk 


\section{Introduction}

Much of human activity entails delegation, both at the inter-personal level and at the societal level. One reason for delegating decisions is that while we may have clear preferences for certain options over others, we often lack the ability or authority to implement such choices. In addition, given the difficulty of aggregating heterogeneous preferences, delegation has the potential to ease decision-making. Hence, in many situations, we rely on a "representative," such as a family member or a politician, depending on the scale of the decision task. At the same time, we express preferences with varying degrees of formality; examples range from voting on political candidates or referendum topics in democracies to letting a member of the household choose where to have dinner.

Many group decisions involving the provision of a public good rely on voluntary contributions, which are beneficial for the group, but costly for individuals. Here, we focus on the provision of a threshold public good, which is worthless unless enough has been contributed, either because the scale of the project requires a minimum investment, or because of its non-scalability. Examples include the construction of a dam, national defense investments, biodiversity conservation, freshwater eutrophication prevention and efforts to mitigate dangerous $\mathrm{CO}_{2}$ concentration levels. Since often delegates make these decisions, we look at the interplay between delegation and threshold public goods provision. Delegation could potentially play a big role here because, unlike linear public goods games where the dominant strategy is to defect, threshold public goods games have a second equilibrium entailing provision, and thus require coordination among decision makers. One may thus expect delegates, insofar as they have information about the preferences of their voters, to be better positioned for coordinating on the preferable equilibrium when negotiating with other delegates than a group where individuals represent themselves. We aim to test such conjecture. Specifically, does this institution induce higher provision, relative to self-representation, or are delegates more prone to pursuing self-interest at the 
expense of the group? ${ }^{1}$ And what role does the constituency play in steering delegates' choices?

We analyze the provision of a threshold public good, which only has value if enough has been contributed collectively, either because the scale of the project requires a minimum investment or owing to its non-scalability. To mimic the challenges to cooperation faced by parties deciding on effort coordination in the presence of uncertainty, all treatments feature both uncertainty on the location of the threshold and on the consequences of non-provision. We test the impact of delegation and peer pressure in the form of payoff-immaterial messages to the delegate by comparing provision levels to a baseline treatment in which subjects self-represent and directly decide on the contribution to the public good. The novelty is the hierarchical structure of the provision choice, as we investigate whether elected delegates deciding on behalf of their constituency are better placed than self-representing individuals (the standard cohort in the literature) in coordinating contributions.

In line with theoretical expectation and with the literature reviewed below, we find that the greater the probability of losses from underprovision, the larger the contributions to the public good, while uncertainty about the location of the threshold induces lower contributions than certainty. Contrary to the prediction of rational choice theory that our delegation treatments should not affect contributions (and in the absence of prior experiments investigating delegation in this setting) our main result is that when delegation is coupled with public pressure, it has a significantly negative effect on contributions. The reason is that delegates tend to adhere to the lowest contribution suggested by the constituency, while disregarding the more cooperative suggestion.

To fix ideas, consider the following two examples of real-world situations characterized by such hierarchical decision-making and avoidance of losses from underprovision. Several nearby condominiums, each comprised of adjacent privately owned houses (or nearby apartment complexes each comprised of multiple flats), need to decide on a joint protection investment to counter rising wildfires in the area. Such

\footnotetext{
${ }^{1}$ Throughout this paper, we refer to a "group" as a society that consists of "teams." Under delegation, each team elects a "delegate", who acts on behalf of the team and shares the earnings equally with her team members.
} 
investment would finance an early warning system or exterior sprinkle system with the aim to deter wildfire damage. To this end, each condominium elects an administrator, who is delegated the decision of how much to invest (on behalf of each household) in joint protection. If the total investments committed by the administrators is below a target sum (the cost of the early warning or exterior sprinkle systems), the project is not provided and all households face a high probability of large losses due to wildfire. If, on the other hand, the target is reached, the loss is avoided with certainty. A more international example concerns EU-wide investments in defense (e.g. a common army or technology): such decision arguably requires delegation and implementation is conditional on reaching an investment threshold; if investments are insufficient, severe losses may be incurred. In such situations there may be considerable uncertainty about the magnitude of investment required to avoid losses, a feature we capture in the lab by manipulating the degree of uncertainty about the threshold.

In this section we couch our contribution in the context of some recent literature pertaining to the role of delegation and (potentially uncertain) contribution thresholds in influencing cooperation (as measured by private provision of public goods). We relegate a longer discussion of related literature to Online Appendix, Section 4.

Much research has studied what facilitates cooperation in experimental settings, notably utilizing public goods games to capture the conflicting strategic motives behind self- and group-interest. The bulk of the experimental literature on public good provision has focused on the linear case, where choices are dominant strategies and the only equilibrium entails zero contributions to the public good. The first strand of experimental literature that we contribute to studies instead the provision of threshold public goods games, where, as in linear games, each group member decides what fraction of the endowment to contribute to a public good. The difference is that only if the group contribution exceeds a threshold, the public good is provided and each player receives a fixed payment, independent of individual contributions. Otherwise, contributions are typically wasted (unless refunded; Croson and Marks, 2000 review a large body of such experiments). Even in the presence of a known threshold, coordination can be difficult, especially when the parties have different stakes in the game (Tavoni et al., 2011). Threshold uncertainty also undermines cooperation: subjects dealing with an uncertain 
common loss arising from crossing a threshold whose location is uncertain find it more difficult to sustain cooperation, compared to those who face a certain provision threshold. Uncertainty on the location of the tipping point thus aggravates the coordination task, increasing the tendency to slip into inaction (Barrett and Dannenberg, 2012; Dannenberg et al., 2015), while increased probability of loss if the threshold is missed unsurprisingly facilitates coordination by inducing higher contributions to the public good (Milinski et al., 2008). We revisit this literature by manipulating both the probability of the loss associated with underprovision, and uncertainty on the location of the threshold.

Arguably, decisions on the provision of threshold public goods, whether they entail investing in security, building a dam or submitting a national mitigation plan as part of an international effort to avoid dangerous climate change, are typically delegated to local or national representatives. Yet, the few existing experiments that study the effect of electoral delegation on the provision of public goods are not concerned with coordination (but rather with cooperation). Hamman, Weber, and Woon (2011) analyze the effect of delegation in a linear public goods game, where nine players in a group choose a single allocator who decides the amount of contributions for all players. They find that most groups elect high contributors who choose maximum contributions on behalf of all group members including themselves. In a recent study, Kocher, Tan and Yu (2018) also use a linear public goods game with nine players where delegates decide contributions on behalf of their constituency. The main difference here is that the delegates' decision powers are confined to the local level (i.e. 3-players teams), such that the three elected delegates simultaneously determine contributions to the "global public good". They find that subjects tend to vote for delegates who assign equal contributions for every team member, although heterogeneity in contributions across teams reduces aggregate contributions (and efficiency) over time. To the best of our knowledge we are the first to investigate how delegation affects public good provision choices that are not (always) dominant strategies ${ }^{2}$. We expect delegation to play a bigger role in such a coordination game, where a second

\footnotetext{
${ }^{2}$ In linear public goods games the dominant strategy is not investing in the public good at all, while the threshold public goods game we use, due to the non-linearity introduced by the threshold, entails different strategies depending on what one assumes about the other delegates' contributions. If the threshold is perceived to be out of reach, we are back in the above case; if instead the threshold is within reach, provision choices can either be strategic substitutes (if the decision-maker does not consider herself pivotal for reaching the target investment) or complements otherwise.
} 
equilibrium entailing public good provision exists and players can free-ride on others' provision, compared to the linear case. The reason is intuitive: delegates may be better positioned to coordinate on the preferable equilibrium than self-representing individuals, insofar as information about their constituencies' preferences is valuable. Our experiment is designed to test this intuition. ${ }^{3}$

To this end, our experimental treatments are designed to assess how the type of constituency that delegates are responsible to, and the degree of public pressure they are exposed to, affects the provision of a threshold public good. We tackle these issues in the laboratory, where we randomly assign twelve subjects (the society) into four teams of three players, and ask each team to elect a delegate through the mechanism of majority voting.

In both treatments the four locally elected delegates move to a global negotiation room where they independently and simultaneously submit the contribution to the public good on behalf of their team. However, while in treatment "DnoM" (Delegation without messages) they only interact with fellow delegates, in "DM" (Delegation and messages) delegates make decisions under the scrutiny of their constituency, i.e. they are joined in the negotiation room by their two team members, each of whom signals his preferred contribution level with a non-binding text message. While the design abstracts away from real-world complexities inherent in the delegated provision of public goods, it captures the frequently observed hierarchical structure of decision-making. See Figure 1 for schematic representation of our delegation treatments.

$<<$ Figure 1 Here $>>$

In addition to the use of a threshold public goods game instead of a linear one, three design features set the present experiment apart from the most closely related literature (e.g. Hamman, Weber, and Woon, 2011; Kocher, Tan and Yu, 2018). First, the focus of our main treatments is what one might call "minimalist delegation", absent any concern for re-election on the part of delegates. Second, we don't aim to assess exploitative behavior

\footnotetext{
${ }^{3}$ A second reason for the importance of delegation in our threshold public goods experiment is that, as argued in footnote 2, and unlike in the linear setups employed by Hamman, Weber, and Woon, 2011 as well as Kocher, Tan and $\mathrm{Yu}, 2018$, provision choices can be strategic substitutes. This implies that the constituency may have an incentive to strategically delegate to agents who have a lower valuation for the public good than they have themselves, in order to free-ride on the others' provision (Habla and Winkler, 2016). While testing for strategic delegation is not the goal here, it is interesting to see whether we observe such pattern in the election of delegates.
} 
by delegates within their teams (which could arise if they were free to impose a higher burden on their own constituency), but focus instead on the effect of the delegation institution in coordinating contributions between teams. As a corollary to our interest in inter-team coordination mediated by delegation, our matching groups entail a larger number of teams (four) than in the literature, to better capture the challenge of avoiding mis-coordination. We thus abstract from electoral concerns and minimize other strategic motives within teams, as follows. The elected delegates' tenure lasts for the entire experiment (without further votes), such that they play several variants of a one-shot public goods game in which losses can ensue if the sum of their contributions falls short of a threshold. Earnings are split evenly among the team members. Consequently, the main outcome variable we aim to capture is the group-wide contribution level (relative to the provision threshold), resulting from different local institutions at the team level (selfrepresentation vs delegation) and different degrees of pressure on delegates. ${ }^{4}$

Beginning with Charness, Rigotti and Rustichini (2007), a related literature developed that focus on the salience of group membership in driving individual behavior in coordination and cooperation games. Charness, Rigotti and Rustichini (2007) focus on the performance of groups relative to individuals in playing two games (battle of the sexes and prisoner's dilemma), by assessing the effect of group membership, which is conveyed in the lab by letting a player's own group watch as a "passive audience as decisions are made" by a randomly selected group member. They find that this way of making group membership salient increases the aggressiveness of the decision-makers under scrutiny. While our setup is widely different, we also find that scrutiny by team members, who observe the delegate and each send her a message signaling their preferred contribution, tend to push the delegate away from cooperation. Specifically, we find that when delegation is coupled with public pressure, it has a significantly negative effect on contributions, despite the fact that by design the delegates are free to choose their preferred public good contribution, and could in principle disregard their constituency. The reason

\footnotetext{
${ }^{4}$ Note that the threshold public goods game in our experiment encompasses both rounds of play where coordination on threshold provision is individually optimal, as well as rounds in which the typical cooperation dilemma arises (i.e. one is better off not contributing). However, our design is not necessarily representative of other types of strategic interactions, such as those occurring when individuals access common property resources characterized by rivalry in extraction. See Stoop, Noussair and Van Soest (2012) for a comparison of recreational fishermen's behaviour in the lab and in the field.
} 
is that delegates appear to give more weight to the least cooperative message: they focus on the lower of the two public good contributions suggested by their teammates. ${ }^{5}$

In Section II, we briefly present the model underlying the game tested here. Sections III-V are respectively concerned with design, experimental results and discussion, followed by a brief conclusion in Section VI. Main supporting tables and figures, additional discussion on social motives, theoretical results, laboratory materials, literature discussion, as well as additional tables and figures concerning the empirical analysis appear in Online Appendix (at tiny.cc/xkgl8y).

\section{Threshold Public Goods Game}

There are $N$ teams and each has $k$ members. Each member has initial endowment $e$ and, thus, each team collectively has an endowment of $E=k e$. Each team $i$ decides simultaneously how much to contribute as a team, $C_{i} \in[0, E]$, to reach a threshold $T$, and no team can reach the threshold on its own: $E<T$. If the sum of all teams' contributions exceeds the threshold, $\sum_{i} C_{i} \geq T$, then they avoid the potential loss, and each team $i$ enjoys the remaining amount, $E-C_{i}$. Otherwise, each team is left with a share of $q \in[0,1)$ of the remaining amount with probability $p$ (so that with probability $1-p$ it still enjoys the entirety of $\left.E-C_{i}\right)$. There is no rebate: players do not receive their contributions back. The payoff function with certain threshold is written as follows:

$$
g_{i}=\left\{\begin{array}{c}
E-C_{i}, \text { if } \sum_{i} C_{i} \geq T \\
p q\left(E-C_{i}\right)+(1-p)\left(E-C_{i}\right), \text { if } \sum_{i} C_{i}<T
\end{array}\right.
$$

There are two symmetric pure strategy Nash equilibria, namely no contribution (NC) and a symmetric provision contribution (SPC) (i.e., each team contributes $T / N$ ). The second equilibrium exists only if the payoff in SPC exceeds the expected payoff in NC:

$$
E-\frac{T}{N} \geq p q E+(1-p) E \Leftrightarrow p E(1-q) \geq \frac{T}{N}
$$

Thus, given $E, T$, and $N$, SPC exists for sufficiently high probability of loss $p$ and/or damage rate $(1-q)$.

\footnotetext{
${ }^{5}$ Put differently, delegation has the potential to bring groups closer to the non-cooperative equilibrium (where the threshold public good is not provided), due the negative effect of messages on delegate contributions, since the latter are largely driven by the lowest value suggested by the constituency.
} 
In this section, we restrict attention to the comparison of these two symmetric pure strategy equilibria, since they are likely to be focal relative to the many asymmetric equilibria in this game (Cadsby and Maynes, 1999, Dannenberg et al., 2015). Thus, teams' choices are expected to coalesce around those points.

We experimentally study this threshold public goods game both in the presence of a certain threshold $T$, and when the threshold is a random variable $U T$. Under uncertainty about the location of the threshold, one of two equally likely thresholds $T_{1}$ and $T_{2}$ is selected randomly, with mean equal to $T$ (i.e., $E(U T)=0.5 T_{1}+0.5 T_{2}=T$, with $T_{1}<T_{2}$ ). The payoff function with uncertain threshold is written as follows:

$$
g_{i}^{u}=\left\{\begin{array}{c}
E-C_{i}, \text { if } \sum_{i} C_{i} \geq T_{2} \\
0.5\left(E-C_{i}\right)+0.5\left(p q\left(E-C_{i}\right)+(1-p)\left(E-C_{i}\right)\right), \text { if } T_{1} \leq \sum_{i} C_{i}<T_{2} \\
p q\left(E-C_{i}\right)+(1-p)\left(E-C_{i}\right), \text { if } \sum_{i} C_{i}<T_{1}
\end{array}\right.
$$

There are three symmetric pure strategy Nash equilibria with threshold uncertainty: no contribution ( $\mathrm{NC}$, as for the certain threshold), and symmetric provisions aiming to reach either $T_{1}$ (SPC1) or $T_{2}$ (SPC2). In the latter two, each team contributes $T_{1} / N$ and $T_{2} / N$, respectively. The expected payoffs of SPC1 and SPC2 are, respectively:

$$
\frac{1}{2} p q\left(E-\frac{T_{1}}{N}\right)+\frac{1}{2}\left(E-\frac{T_{1}}{N}\right) \text { and } E-\frac{T_{2}}{N}
$$

Hypothesis 1: Total contributions to the public good increase in $p$.

We expect overall team contributions to increase in $p$, since team $i$ 's expected payoff under NC decreases ( 1 and 3), meaning that it would take a much bigger gamble by not contributing under both certain and uncertain threshold cases. An increase in $\mathrm{p}$ also increases attractiveness of SPC2 in comparison to SPC1.

No contribution is the unique equilibrium in a standard public goods game because of free-riding incentives. By introducing the certain threshold, the game is transformed into one involving a choice between $\mathrm{NC}$ and SPC, and decision makers face the well-known problem of coordination owing to strategic uncertainty.

Let $\pi_{i}$ be the subjective beliefs of team $i$ about reaching the threshold when targeting the SPC (contributing $T / N$ ), given the uncertainty about whether total contributions, including those by other teams, will suffice to reach the certain threshold. Assuming symmetry, team $i$ believes that if it contributes less than $T / N$, the other teams' 
contributions will not suffice to reach the certain threshold. Condition (2) can be modified to accommodate subjective beliefs, as follows:

$$
\begin{gathered}
u\left(T / N \mid \pi_{i}\right) \geq u\left(0 \mid \pi_{i}\right) \Leftrightarrow \\
\pi_{i}\left(E-\frac{T}{N}\right)+\left(1-\pi_{i}\right)\left(p q\left(E-\frac{T}{N}\right)+(1-p)\left(E-\frac{T}{N}\right)\right) \geq p q E+(1-p) E \\
\Leftrightarrow \pi_{i} \geq \frac{T(1-p(1-q))}{(E N-T) p(1-q)}
\end{gathered}
$$

where $u\left(C_{i} \mid \pi_{i}\right)$ is team $i$ 's (expected) utility given its subjective beliefs about reaching the threshold when contributed $T / N$. The threshold will be met provided that the average contribution of others is at least $\mathrm{T} / \mathrm{N}$ (thus, $\sum_{\mathrm{j} \neq \mathrm{i}}^{\mathrm{N}} \mathrm{C}_{\mathrm{j}} \geq \frac{\mathrm{T}(\mathrm{N}-1)}{\mathrm{N}}$.) However, team $i$ places probability $\left(1-\pi_{i}\right)$ on the event that the average contribution of others is lower than $T / N$ (thus, $\sum_{j \neq i}^{N} C_{j}<\frac{T(N-1)}{N}$.) Thus, introducing subjective probability impedes coordination, as teams are less willing to aim for the symmetric provision equilibrium with subjective probability in equation (5) than without it in equation (2).

Note that uncertainty is involved in both symmetric equilibria (NC and SPC) when $\pi_{i}$ is included. While the expected payoff under no contribution depends only on $p$, the expected payoff under the symmetric provision equilibrium depends on both $p$ and $\pi_{i}$. Thus, in choosing between the two strategies, teams may opt for the "safer" zero contribution strategy. ${ }^{6}$

Hypothesis 2: Total team contributions are lower under threshold uncertainty than under certainty about the location of the threshold

Two possible reasons for the total team contributions to decrease under threshold uncertainty are i) general tendency for the decision-makers to have risk-averse preferences, captured by some concave utility functions and ii) the possibility of coordination failure. While the first is a commonly known, let us expand the discussion on the second more below.

Let $\pi_{i}^{1}$ and $\pi_{i}^{2}$ be the subjective beliefs of team $i$ about reaching the threshold when targeting SPC1 and SPC2 (contributing $T_{1} / N$ and $T_{2} / N$, respectively). These beliefs are

\footnotetext{
${ }^{6}$ This game resembles the stag-hunt game in which players have to decide between socially cooperative and safe strategies. By contributing positive amounts, a team runs the risk of becoming the "sucker" of the group, which lowers the attractiveness of the SPC.
} 
affected by uncertainty about whether total contributions (including those by other teams) will suffice to reach the low and high thresholds, respectively. Assuming symmetry, team $i$ believes that if it contributes less than $T_{1} / N$ or $T_{2} / N$, the other teams' contributions will not suffice to reach the low or high thresholds, respectively. Consequently, miscoordination in reaching either threshold is more likely than with a certain threshold, since contributions can be wasted in two different ways. First, each team contributes $T_{1} / N$ and succeed to reach SPC1. Yet, the threshold can still be $T_{2}$ with probability 0.5 . Second, each team contributes $T_{2} / N$ and succeed to reach SPC2. Yet, the threshold can still be $T_{1}$ with probability 0.5 . These possibilities of wasting contributions lower the expected payoffs of SPC1 and SPC2 given the subjective beliefs $\pi_{i}^{1}$ and $\pi_{i}^{2}$, which makes NC more appealing in comparison to the certain threshold. Therefore, threshold uncertainty is expected to lower contributions.

However, notice that under risk-neutrality and for some fixed parameter values, especially for very high subjective probabilities $\pi_{i}^{2}$ (i.e., each team $i$ has almost no concern about not reaching $T_{2}$, once it contributes $T_{2} / N$ ), payoff under SPC2 can be higher than SPC1 and NC. Since contributions under SPC2 are higher than SPC (i.e., $T_{2} / N>T / N$ ), threshold uncertainty may increase the total team contributions.

\section{Experimental Design}

The experiment was conducted with undergraduate students using a pen and paper protocol at Sogang University, Korea, in February 2013. ${ }^{7}$ The experiment consists of three treatments, with eight independent groups in each treatment, and four teams in each group. In every session, we randomly form $N=4$ teams of variable size $k$ (either one or three team members), which remain unchanged throughout the session. Each team is endowed with $E=120$ laboratory dollars for every round. ${ }^{8}$ Subjects interact anonymously throughout the experiment by indicating their choices on sheets of paper that are distributed

\footnotetext{
${ }^{7}$ Sogang University did not yet have an experimental laboratory at the time we ran our experiment, so we devised a simple paper and pencil protocol which worked seamlessly, and whose implementation was facilitated by RAs and a text messaging application that ensured that the treatments took place in a controlled environment. Furthermore, group identity was important for treatments 2 and especially 3 , as explained below, and our design is arguably more effective for this purpose than a standard computer lab.

${ }^{8}$ To keep the earnings and, thus, the monetary incentives unchanged between treatments, we set the exchange rate between the laboratory dollar and the USD in the first treatment at 3 laboratory dollars $=1$ USD. In the other treatments, the exchange rate is 1 laboratory dollar $=1$ USD. Thus, in all treatment decision makers (self-representing individuals or delegates) allocate 120 laboratory dollars.
} 
and collected by the experimenters. The collected information is shared with the subjects, when necessary, by means of projectors. In the beginning of the session, subjects are warned that they are not allowed to communicate with others throughout the experiment, with the exception of the structured written communication introduced in Treatment 3 (see below). Subjects were always supervised by at least one lab assistant and no attempt of breach of conduct was detected.

\section{A. Game phases}

Each session consists of two phases, namely the practice phase (one round) and the actual game phase, which consists of six one-shot rounds that feature different values of $p$ and threshold uncertainty. Subjects are informed about the two phases and about the fact that one randomly selected round from the actual game phase will determine their earnings at the end of the session. However, subjects did not know the exact number of rounds and the parameters values shown in Table 1 until they played a given round. In each round, the decision makers play a one-shot threshold public goods game. Here, the four teams decide simultaneously on how much to contribute to the public good (in multiples of 1 laboratory dollar) to reach a group threshold $T=240$ laboratory dollars (or the corresponding uncertain and equally likely thresholds, $T_{1}=190$ or $T_{2}=290$ ). Subjects are made aware that failing to collectively reach the threshold means losing $90 \%$ of their remaining endowment (i.e., $q=0.1$ ) with probability $p$. The degree of loss uncertainty is parameterized with three values of $p \in\{0.55,0.75,0.95\}$, hence, ranging from highly uncertain (55\% probability) to almost certain (95\% probability) losses under nonprovision. Thus, subjects face a potential catastrophic outcome based on $q=0.1$ and $p$ values. While we want a low value for $q$, we particularly chose a non-zero value to avoid potential discontinuous behavior around $q=0$. Then, for $q=0.1$ we chose the lowest value of probability $p$ so that expected payoffs are the same for NC and SPC.

\section{$<<$ Table 1 Here $>>$}

In each of the first three rounds, they play the game with certain threshold $T$ and probabilities $p$ equal to $0.55,0.75$, and 0.95 . In addition to the varying degree of loss uncertainty captured by the three values of $p$, the following three rounds are characterized by uncertainty over the location of the threshold UT. Here, $T_{1}$ and $T_{2}$ have equal probability of materializing, and the mean of the two thresholds is 240 , as in the first three 
rounds featuring threshold certainty. Note that the game phase (i.e., the financially incentivized phase) is designed such that over the course of the six rounds, the teams face each value of $p$ twice, once for certainty and once for uncertainty over the location of the threshold. We chose the game played in practice round so that subjects experience the most complicated version before playing the incentivized rounds. ${ }^{9}$ Also, we chose the order of threshold (un)certainty in the game phase so that the subjects deals with similar problems in a row. The order of probabilities played in 6 rounds are determined randomly before the experiment, without replacement, and with the same order of rounds played in every session. $^{10}$

Table 1 summarizes the main parameters used in the experiment and the accompanying symmetric pure strategy Nash equilibrium attaining the highest expected payoff. Note that in all rounds, NC is always an equilibrium, but depending on the two types of uncertainty, provision can also be an equilibrium. Round 5 is designed to trigger the least contributions to the public good, as the only equilibrium is the no contribution case. Round 2 is next, as risk neutral players will be indifferent between NC and SPC. In the remaining rounds, public good provision is the Pareto-superior equilibrium. The difference between Rounds 1 and 3 and Rounds 4 and 6 is that, in the latter two, which feature threshold location uncertainty, the highest payoff is attained when the highest and lowest targets are met in Round 4 and 6, respectively. Notice that as $p$ increases, the highest expected payoff equilibrium becomes the one with higher contribution as predicted in hypothesis 1. Also, by introducing threshold uncertainty, the highest expected payoff equilibrium becomes the one with lower contribution, comparing round 2 with round 5 and round 1 with round 6 as predicted in hypothesis 2 . Threshold uncertainty seems to increase contributions (from SPC to SPC2) when we compare round 3 with round 4. However, in Table 1, the highest expected payoff equilibrium does not take concerns for coordination

\footnotetext{
${ }^{9} \mathrm{We}$ asked several control questions before the practice phase took place, to make sure that all subjects understood the game. We did not start the experiment until every subject had answered correctly all questions. The instructions, control questions, decision sheets, and survey questions can be found at https://goo.gl/tgSIHr.

${ }^{10} \mathrm{We}$ are going to control the order of play in empirical analysis. Similarly, we are going to discuss the impact of the specific game played in practice phase on decision makers as it might produce more noncooperative behaviour in Section IV.
} 
failures (subjective probabilities assumed to be equal to one) and risk preferences into account, which would both decrease contributions as discussed earlier.

To facilitate the coordination task, we also introduce a pledge stage in each round before subjects decide their contributions. In the pledge stage, they announce how much they intend to contribute and how much they expect the other teams to contribute. Then, they play the contribution stage, in which they input their actual contributions to the public good.

We announce the other teams' contributions and results of each round at the end of the session to minimize possible income effects owing to performance in a given round. We treat each round as independent observation of a one-shot threshold public goods game. Since subjects learn others' pledges in every round, we control round effects and also provide results using only the observation from the first round. Moreover, we randomly allocate anonymous ID cards to subjects to determine teams and to control reputation effects. ${ }^{11}$

\section{B. Practice phase and voting}

After teams are formed, subjects are allocated to their team rooms. The session begins with the unpaid practice phase in which team members play a game within the team with uncertain threshold $U T$ and probability $p=0.55$. Each team member decides how much to pledge, as well as how much s/he expects other teams to contribute. This information is projected onto a screen, together with the subjects' IDs. Team members observe their teammates' pledges and expectations from other teams and decide how much to contribute, which is also projected onto the screen with the subjects' IDs. No information (pledges, expectations, and contributions) are revealed to other teams to avoid learning. The purpose of the practice round is subjects to form an opinion about their teammates' willingness to invest in the public good. It is particularly important for the treatments with $k=3$, in which each team anonymously elects a delegate after the pledge stage (before the contribution stage; see below). In fact, this is the only information about candidates at the moment of voting.

To elect a delegate, every team member is a candidate and can vote for anyone, including him or herself. The majority voting eliminates ties and guarantees that a delegate

\footnotetext{
${ }^{11}$ Details about the ID cards and other experimental procedures are in Online Appendix, Section 4.
} 
is chosen, except in the case in which all subjects have one vote. In this case, another vote takes place to determine the least wanted candidate. Again, majority voting eliminates ties and one team member is chosen to not to be a delegate, except in the case of again all having one vote. In this case, we randomly eliminate one candidate. Majority voting then guarantees a delegate is chosen with two candidates and three votes. Random elimination was only needed 3 times out of 64 elections.

\section{Treatments}

Treatment 1: No delegation (NoD): Individual "delegates" represent only themselves, play six rounds, and receive all the team's earnings. ${ }^{12}$

Treatment 2: Delegation, no messages (DnoM): The elected delegates of the teams move to another room to play all six rounds. The delegates decide how much their team will contribute without communicating with their teammates. In each round, the information about the pledge stage is also revealed to the non-delegates (i.e., four teams' IDs and each team's pledge and expectations from others) and they are asked for their opinion on how much their delegate should contribute. Note that teammates could recognize their delegate's pledge and their expectations regarding other teams, as they know their team's ID. So, teammates have the same amount of information as their delegates before deciding how much their team should contribute. The earnings of the team are split evenly among the members.

Treatment 3: Delegation and messages (DM): In contrast to DnoM, in DM, all elected delegates and the non-delegates move to a common room. The delegates are seated in the first rows and their teammates just behind them. Teammates do not see their delegate's decision sheet, but the delegate feels the pressure of his or her teammates sitting behind. All subjects know who the delegates are, since they sit in the front rows, and the nondelegates as they sit behind their delegates. Round 1 begins with the pledge stage in which delegates represent their teams and the pledges are declared to all (i.e., four teams' IDs and each team's pledge and expectations from others). Teammates could recognize their delegate's pledge and their expectations regarding other teams, as they know their team's ID. So, teammates have the same amount of information as their delegates before deciding

\footnotetext{
${ }^{12}$ For the summary of treatments, see Online Appendix, Table 1.
} 
how much their team should contribute. After the pledge stage, each non-delegate sends his opinion about how much the team should contribute to the team's delegate via the experimenters. The delegate checks the teammates' opinions and freely chooses the team's contribution level. Furthermore, recall that the delegates' contributions are not revealed until the end of the session. The remaining rounds follow the same procedure. Finally, the earnings of the team are split evenly among the members. ${ }^{13}$ Notice that there are two main differences between DnoM and DM treatments: teammates presence and team communication. Therefore, any difference between these two treatments could be a result of any or joint effect of the two features.

In the experiment, we design the delegate and her teammates to have the same objective function and information. Furthermore, as the team's earnings are also shared equally among the members, there is no strategic interaction between them. Incorporating these design features to the model discussed above (e.g., with standard linear or concave utility functions), the delegate's behavior would be the same as that of a self-representing individual player, irrespective of whether communication within teams is allowed. ${ }^{14}$

Hypothesis 3: When delegates do not receive messages from their team members they contribute on average the same as self-representing individuals.

Hypothesis 4: When delegates receive messages from their team members they contribute on average the same as self-representing individuals.

Notice also that the hypotheses 3 and 4 imply that delegates respond to the changes in $p$ and threshold uncertainty same as the self-representing individuals, irrespective of whether communication within teams is allowed. Relatedly, similar experiments with selfrepresenting individuals find total individual contributions to increase in $p$ (Milinski et al., 2008), and decrease in threshold uncertainty (Barrett and Dannenberg, 2012; Dannenberg et al., 2015) in line with our hypotheses 1 and 2.

\section{Empirical Results \\ A. Random assignments of subjects}

\footnotetext{
13 The average earnings were $10,195 \mathrm{KRW}$, with a maximum of 40,000 and a minimum of 1,000 . Additionally, subjects receive the show-up fee of 10,000 KRW (about 10 USD).

${ }^{14}$ Furthermore, as we explain in Section III, we rule out strategic communication between teams.
} 
224 subjects took part in the experiment. 32 subjects in the noD treatment and 96 (32 delegates and 64 non-delegates) in each of the DnoM and DM treatments. We collected basic background information (e.g., age, gender, and major,) as well as some attitudinal variables (e.g., attitudes toward general risk, the lottery, and global warming) and thoughts about their choices during the experiment via post-experiment surveys. ${ }^{15} 56-62$ percent of subjects are male, and the average age is $22-23$. On average, they are enrolled for about five semesters, and approximately 60 percent of the students are economics or business majors. We find no significant differences in any background and also attitudinal variables across treatments. This suggests that subjects are assigned randomly to observables across treatments.

\section{B. Group contribution}

For the purpose of our study, a key outcome is the total contribution of a group. Figure 2 shows the average group contribution (there are eight groups per treatment) by treatment, disaster probability, and threshold uncertainty. ${ }^{16}$ There are three notable results. First, as the probability of loss increases (from $55 \%$ to $95 \%$ ), the group contribution tends to be larger. This is not surprising, since the incentive to avoid the risk of the loss should be larger when the probability of loss is higher. Second, comparing the average group contribution between $\mathrm{T}$ and UT for each probability and treatment, we find that participants tend to contribute less when they face uncertainty about thresholds. Lastly, we find that the average group contribution is the largest in the baseline treatment (noD), and smallest in the DM. Next, we compare the average group contribution by treatment for each probability and threshold uncertainty setting (T or UT).

$<<$ Figure 2 Here $>>$

We conducted Kruskall-Wallis tests to compare the average contributions across conditions. Average contributions across all 18 conditions are different and the differences are statistically significant at the $1 \%$ level according to the Kruskall-Wallis test result. We also conducted the test across three treatments (noD, DnoM and DM), by whether the

\footnotetext{
${ }^{15}$ See Online Appendix, Table 2.

${ }^{16}$ Further results, including contributions in each group, pledges and survey findings are contained in Online Appendix, Section 6.
} 
threshold is certain or uncertain, or by the probability of loss. The differences are all statistically significant ( $\mathrm{p}$-value is $0.007,0.043$, and less than 0.001 , respectively).

We also confirm the above findings from unconditional mean differences by regression analysis. We run the following regression at the group level:

$$
C_{j r}=\beta_{0}+\beta_{1} \text { Dno }_{j}+\beta_{2} D M_{j}+\gamma_{1} p 75_{r}+\gamma_{2} p 95_{r}+\gamma_{3} U T_{r}+\epsilon_{j r},
$$

where the dependent variable $C_{j r}$ is the total contribution made by group $j$ in round $r=$ $1, \ldots, 6$. $\operatorname{DnoM}_{j}$ (or $D M_{j}$ ) indicates whether the group belongs to treatment DnoM (or DM). The omitted treatment is noD. Then, $p 75_{r}\left(\right.$ or $\left.p 95_{r}\right)$ indicates whether the probability of loss is $75 \%$ (or 95\%) in round $r$. The omitted probability is $55 \%$. Lastly, $U T_{r}$ indicates whether the threshold in round $r$ is uncertain.

The results in Table 2 confirm our findings in Figure 1. Column 1 shows the results for all group-round observations. First, compared to noD, the group contribution in DnoM is lower by about 17 lab $\$$. This gap is not statistically significant. However, the group contribution is significantly lower in DM, by about 51 lab $\$$. This pattern by treatment holds after we separate the sample by the probability of loss, from Columns 2, 3, and 4, although the statistical significance differs. Therefore, we cannot reject the hypothesis 3 (i.e., delegates and self-representing individuals contribute the same on average when delegates do not communicate with their team members), but reject the hypothesis 4 (i.e., delegates and self-representing individuals contribute the same on average when delegates do communicate with their team members.)

$$
<<\text { Table } 2 \text { Here }>>
$$

One may be concerned that the experimental design induces a learning effect, since subjects play multiple rounds in a fixed group, and might be influenced by previous rounds or due to dynamic interaction among players in a group. To address this concern, in the last column, we present the results using only the first round for each treatment, which is the one featuring T75\%. The number of observations is reduced to $24(=8$ groups $\times 3$ treatments). However, the group contribution remains significantly lower in DM than in noD.

Another concern mentioned in footnote 10 is that the game played in practice phase might have an anchoring effect and produces more non-cooperative behavior in both DnoM and DM. This possible effect should lead different to average contribution levels between 
NoD and DnoM. However, we find no significant difference. At the same time, this effect should not affect the behavior between DnoM and DM, since it is present in both treatments. Thus, lower delegate contributions with team member communication should not be driven by the game played in the practice phase.

Second, the group contribution is significantly higher, by 62 lab $\$$, when the loss probability is $75 \%$, and even higher, by 101 lab $\$$, when it is $95 \%$. Thus, we cannot reject the hypothesis 1 (i.e., Total contributions to the public good increase in $p$.) Lastly, when the threshold is uncertain, the total group contribution is lower. This result is in line with our theoretical finding that threshold uncertainty should deepen the coordination failure. The effect of threshold uncertainty increases with the probability of loss. Thus, we cannot reject hypothesis 2 (i.e., total team contributions are lower under threshold uncertainty than under certainty about the location of the threshold.)

\section{Team decisions}

We find that the total group contribution is lower when decisions are made by delegates (in DnoM and DM), and particularly when delegates are informed of their team members' opinions (in DM). To explain this finding, we examine the individual team decisions, as well as how the experimental and contextual variables affect decision makers in the different treatments. Specifically, we estimate the following equation, which determines the individual team choices:

$c_{i j r}=\beta_{1} P l d g_{-i j r}+\beta_{2} \operatorname{Exp}_{-i j r}+\gamma_{1} p 75_{r}+\gamma_{2} p 95_{r}+\gamma_{2} U T_{r}+M_{i j r} \delta+\alpha_{i j}+\tau_{r}+\epsilon_{j r}$,

where the dependent variable, $c_{i j r}$, is the contribution of team $i$ in group $j$ in round $r$, and $P l d g_{-i j r}$ and $E x p_{-i j r}$ represent the average pledge and the expectation presented by the other teams in the same group, respectively. Then, $M_{i j r}$ encapsulates the messages from the team's non-delegates, and, as a value, either takes the average of the two messages, or takes the higher or lower of the two messages. ${ }^{17}$ Lastly, we include individual-team fixed effects $\alpha_{i j}$ and round-specific fixed effects $\tau_{r} .{ }^{18}$ The individual-team fixed effects control

\footnotetext{
${ }^{17}$ Note that $M_{i j r}$ cannot be included for noD since there is no delegation in this treatment. Non-delegates' opinions are not delivered to delegates in DnoM. Therefore, there should be no effect of non-delegates' opinions on delegates' decisions in DnoM. This will serve as a validity test of our experimental design.

${ }^{18}$ For round-specific fixed effects, although there are six rounds, we can only include three dummies owing to perfect linear collinearity with $p 75_{r}, p 95_{r}$, and $U T_{r}$.
} 
for any effects from each team's time-invariant characteristics, including delegates' demographic characteristics or specific traits, as delegates are fixed once they are determined by voting. In addition, the round-specific fixed effects control for any potential dynamic effects over rounds. Therefore, $\delta$ is identified off the variation in teammates' messages round by round.

\section{$<<$ Table 3 Here $>>$}

Table 3 presents the regression results. First, it is notable that there is no effect from average pledges or from the expectations of other decision makers. This appears to suggest that, in accordance with the model studied in Section II, but in contrast to field and experimental evidence, communication does not facilitate coordination in this game. Second, the probability of loss significantly affects teams' contributions.

Comparing Columns 1, 2, and 5, we find that the effects have similar magnitudes across treatments. Lastly, we find that threshold uncertainty negatively affects the team contribution in DM only. In other words, delegates contribute less when the threshold is uncertain only if they receive their teammates' messages.

Instead of controlling for individual decision maker fixed effects, we control for the decision makers' characteristics and check how the characteristics affect the level of the contribution. ${ }^{19}$ Overall, the results for the experimental control variables are similar to those in Table 3. What is interesting is that decision makers' individual characteristics, such as gender, major, and risk aversion, matter in the noD and DnoM treatments, but are statistically insignificant in DM. One interpretation is that with non-delegates' opinions available, delegates behave less as individuals and more as anonymous representatives of a team..$^{20}$

\section{The influence of non-delegates' messages}

In Column 6 of Table 3, we find that delegates tend to follow the average of the non-delegates' messages. Column 7 shows the main finding of this paper, i.e. that the negative effect of messages on contributions is largely driven by the lower of the two values

\footnotetext{
${ }^{19}$ The full results are presented in Online Appendix, Table 3.

${ }^{20}$ Furthermore, the results about the age effect are intriguing. In noD, age does not matter, while it has a positive effect in DnoM, where older delegates contribute more. However, the effect is opposite in DM; older delegates contribute less after controlling for non-delegates' opinions. While interesting, one should be cautious about inferring from these results, given that there is little age variation among the subjects.
} 
suggested by the constituency. The higher message does not have any significant impact on delegates' decisions: delegates appear to concentrate on the non-cooperative suggestion, ultimately leading DM groups to gravitate towards the $\mathrm{NC}$ equilibrium ${ }^{21}$. We find similar results, except that the higher opinion is also significant, but matters less than the lower opinion. As expected, in Table 3, it turns out that teammates' messages do not matter in DnoM. This is not surprising in that the messages are not delivered to delegates. On the other hand, the result indicates that the significant effects of the messages in DM are not driven by unobservable confounders that could affect both delegates and non-delegates, even with no communication between them. ${ }^{22}$

Furthermore, it is notable that delegates are particularly sensitive to non-delegates' zero contribution messages. We find that in 93 of the 192 choices, at least one non-delegate suggested a zero contribution and, in 63 of the 93 choices (68\%), delegates actually contributed zero. Obviously, we find no effect of non-delegates' opinions in DnoM. However, the effect of the lower message is not solely driven by the zero contribution messages. In Column 8 of Table 3, we restrict the sample to the cases where the lower message is not zero. We still find that delegates are influenced by the lower message, but not significantly by the higher message.

Lastly, we check the robustness of the finding that delegates are more sensitive to the lower contribution message across various subsamples. ${ }^{23}$ Overall, we find that our main findings are robust. We find that delegates are more inclined to the lower message when they do not know anyone in the session or in their team (all strangers). The asymmetric effect is also evident, regardless of whether they understand the game rules well and

\footnotetext{
${ }^{21}$ Note that this result is robust to the exclusion of rounds 2 and 5 , where the payoff from SPC is not strictly greater than the payoff from $\mathrm{NC}$, and hence it is rational to follow the lower message (see Table 1). That is, the results of Table 3 hold after excluding rounds 2 and 5: average message and lower/higher messages do not matter in DnoM in columns (3) and (4), but they are significant in DM in columns (6) and (7). Note also that the model in column (8) restricts the sample to round 1 only, and confirms the result in the "cleanest" setting (devoid of this and other confounders such as contamination across rounds).

${ }^{22}$ We examine both delegates' contributions and non-delegates' messages for all 192 choices (32 delegates $\times 6$ treatments) in DM. Of all 192 choices, $55 \%$ are closer to the lower opinion, while only $21 \%$ are closer to the higher opinion. See Online Appendix, Figure 1.

${ }^{23}$ For the full results, see Online Appendix, Table 4. More specifically, we used the specification of column (7) in Table 3 for various subsamples. For example, there are 192 observations in DM, and 126 are male delegates and 66 are female delegates. For other subsamples, we use information collected by postexperiment surveys (the first four subsamples), by experimental design (single certain threshold or double uncertain thresholds), or by information on pledges and expectations (the last four subsamples).
} 
regardless of gender. The asymmetric effect is not affected by the average pledge or the expectation presented by the other delegates. An interesting finding is that the sensitivity to the lower suggestion is not statistically significant when the threshold is uncertain, although the effect of the lower suggestion is much larger than that of the higher one.

$$
<<\text { Table } 4 \text { Here }>>
$$

Table 4 summarizes the non-delegates' opinions in DnoM and DM by round. The results for DnoM are quite surprising. Although the non-delegates' contribution decision is payoff-irrelevant, we find little difference between non-delegates and delegates. Nondelegates appear to be willing to contribute more, but that is not always the case. ${ }^{24}$

\section{Discussion}

Our finding that delegates contribute less than self-representing individuals, especially when non-delegates' messages are close to zero, is puzzling from the perspective of the model studied in Section II, given that in our setting the delegates and their teammates have the same objective function and there should be no strategic interaction between them. In this section we discuss some possible explanations for our findings about the behavior of delegates. Among others, we focus on two explanations; a) responsibility towards teammates, and b) social regret. Additionally, we investigate the potential role of strategic selection of delegates in Online Appendix Section 2, which we conclude that it is unlikely that selection into delegates itself, or strategic delegation, explains our findings.

Differently from the case of self-representation, a delegate may feel responsible towards her teammates, since her decision affects not only her own payoff but also theirs. We speculate about three reasons why such responsibility could push delegates to contribute less than self-representing individuals. First, delegates may fear to let teammates down and this could induce them to become more cautious, i.e. minimize the risk of being the sucker by gravitating towards NC. Such fear of being blamed for wasting positive contributions could deter investments in the public good even in the absence of communication. Second, in the treatment featuring messages, delegates may feel the need

\footnotetext{
${ }^{24}$ Recall that, unlike in DnoM, non-delegates in DM can signal their preferred contributions to their delegates. However the results in Table 5 show that non-delegates in DM did not behave differently from those in DnoM. Indeed, the last column shows that we cannot reject the null that non-delegates in DM are the same as those in DnoM. This means that the lower public good contributions by the delegates in DM are attributable to the fact that they responded to the messages sent by their constituency.
} 
to conform to the suggestions of their constituency, even if the messages are non-binding, possibly due to a sense of responsibility for having been voted "into office" (in most cases by at least one of the teammates). Combining the two lines of thought above, one would expect the delegate to give more weight to the lower of the two messages about preferred contributions. Third, if a delegate expects other delegates to contribute little to the public good, again due to the above arguments, then she would have an additional incentive not to contribute. Bolton et al. (2015), Charness and Jackson (2009), and Song (2008) suggest similar implications for responsibility and show that delegates trust and cooperate less in trust and stag-hunt games, and choose safer lotteries.

Although we observe a similar tendency, the gap between group contributions in DnoM and NoD was not statistically significant. Messages appear to be determinant in triggering significant reductions in contributions. We thus come back to the interesting case where the delegate receives asymmetric contribution suggestions, e.g. one high and one low message in our setting, and elaborate on its implications. We focus on two reasons why delegates may be more likely to follow low contribution suggestions than high ones. First, the anticipated pain of regret for making the wrong choice is strengthened if people are aware of the foregone choice (Zeelenberg, 1999). Thus, the above responsibility effects are more salient when the delegates receive the teammates' messages. Second, a delegate who receives a low contribution suggestion could easily anticipate that the other delegates also receive and follow such suggestions. Thus, she would also contribute nothing, anticipating that the group would fail to reach the threshold regardless of her contribution. In a lottery choice setup, Bolton et al. (2015) show that delegates tend to conform to both teammates suggesting safe and risky options, but that only the "safe" suggestions have a significant effect. Our experimental data aligns with this finding, in the sense that delegates selectively listen to the message suggesting low or zero contributions (and avoid the risk of wasting contributions, not reaching the target and being the "sucker"). Thus, we argue that some combination of these forces drives the delegates to contribute less than self-representing individuals. $^{25}$

\footnotetext{
${ }^{25}$ Note that, due to the experimental design, we cannot attribute the difference between the DM and DnoM treatments to messages alone. The reason is that while non-delegates were also present in the decision room in DM, they sat in a different room in DnoM. For this reason, we can only conclude that public pressure from
} 
In order to understand the delegate's behavior both in the presence and in the absence of the teammates' messages in the threshold public goods game that we tested experimentally here, we sketch the results of an application of a social regret model (İriş, 2018). The latter theory modifies Loomes and Sugden's (1982) individual regret-aversion model; as detailed in Online Appendix Section 3, we extend İriş (2018) by studying binary choice environments with more than two states of nature. Specifically, this model departs from standard preferences by allowing the delegate to experience regret (or rejoice) for not having followed a teammate's message if the suggested contribution would have secured a higher (lower) payoff. We assume that regret and rejoice are greater for larger utility differences (in an increasing fashion) between what a team receives based on the delegate's contribution and what the same team would have received from another action suggested by the teammates. When the utility difference increases linearly, the delegate is social regret-neutral and acts as if she was maximizing her expected utility. On the other hand, if the utility difference increases convexly, the delegate is social regret-averse. ${ }^{26}$ Social regret-averse delegates value the states with larger utility differences more. In the case of a certain threshold, we have three possible states: reaching the threshold and avoiding the potential loss $(R T)$, not reaching the threshold and suffering the ensuing loss $(N T L)$, and not reaching the threshold, but getting lucky as the loss does not happen (NTNL). Our main result shows that if the utility difference is larger in $N T L$ or $N T N L$ than $R T$, which is satisfied by the parameters chosen in the experiment, then social regret-averse delegates follow low contribution suggestions more than messages calling for high contributions. Thus, social regret-aversion rationalizes the main finding of our experiment.

\section{Conclusion}

As previously mentioned, there are behavioral and theoretical arguments (beyond the realm of rational choice theory) to expect that, relative to the baseline case where decision makers act independently, a delegate who receives contrasting signals by the constituency will place more weight on the non-cooperative messages. In line with this expectation, we find that, while delegation of the investment decision to an appointed

the constituency, in the form of a combination of messages and passive auditing in the decision room, induces the delegates to decrease group contributions to the public good.

${ }^{26}$ See Bleichrodt and Wakker (2015) for more about this evidence and applications of regret theory. 
leader has little effect when decoupled from the peer pressure of non-delegates, it does influence delegate behavior in the treatment featuring public pressure from the constituency. Here the contribution to the public good drops significantly, even if the messages do not alter the delegate's incentives. The empirical analysis confirms that this drop is attributable to the fact that delegates tend to focus on the lowest contribution level suggested by non-delegates. Hence, negative examples can be detrimental to cooperation.

The simple setup utilized here, while arguably more conducive to cooperation than the more complex real-world negotiations, provides stark implications for policy. For example, consider the parallel between the experimental setting and the emissions reduction problem. Reaching an agreement on emission trajectories that are compatible with safe levels of global warming requires collaboration between negotiators (acting on behalf of their national constituencies) and their foreign counterparts. The stakes are indisputably high according to the scientific evidence on the losses associated with substantial warming, such that the collectively rational decision would be to coordinate efforts to avert abrupt future changes in the climate. In the terminology of our game, this is the provision equilibrium, and if all parties do their part, the cost of reducing emissions, relative to business as usual, is more than compensated for by the expected future savings from avoiding dangerous climate changes.

However, as in the game, individual free-riding incentives mean that unilateral deviations from the provision strategy can quickly destabilize cooperation: once a (sufficiently large) country defects, reaching the target may be unfeasible or uneconomical. We can think of this as strategic uncertainty about co-players' actions. The game also captures another feature that has the potential to jeopardize climate cooperation, namely scientific uncertainty on the location of the threshold. As expected, we find that uncertainty reduces the contributions to the public good and, consequently, the probability of coordination on the cautious equilibrium. Lastly, the negative effect of signaling by the constituency points to the dark side of leading by words: delegates appear to be quick to follow suggestions only when these entail pursuing a risky gamble.

Real-life delegation of contribution decisions pertaining to threshold public goods is likely to be more complicated. Here we focused on a simple implementation of the complex political economy context that surrounds delegation; further work may contribute 
to this strand, for instance by disentangling the effect of different forms of public pressure on delegates' contributions. Furthermore, the present setup restricts attention to small groups of symmetric subjects in terms of expected payoffs. Asymmetries entail different stakes for different parties, and a larger group size amplifies the problem of deterring unilateral deviations from the provision strategy. Thus, it appears that, in the face of uncertainty, without a strong call for action at the domestic level, delegates may be reluctant to commit to sizeable contributions and may selectively listen to those who suggest staying with the status quo.

Lastly, it is worth pointing out that the type of interactions captured by this experiment are relevant for more localized cooperation problems such as shared investments in safety, as described in the introduction. Of course, the present design does not shed light on other important cooperation problems, such as the more commonly studied provision of linear public goods. Another specific design choice is the loss frame (decision makers can invest in the public pot in order to avoid a loss): it is likely that a similar experiment framed in the gain domain would induce different behavior. Furthermore different, non-student, subject populations may also behave differently, and even the choice of a different practice phase may have behavioral repercussions, since the dominant strategy here is non-cooperation, potentially priming subjects to defection in later rounds. We hope that the present work will stimulate further experimentation in this direction. 


\section{References}

Barrett, Scott, and Astrid Dannenberg (2012). "Climate negotiations under scientific uncertainty." Proceedings of the National Academy of Sciences 109 (43): 1737217376.

Bleichrodt, Han, and Peter P. Wakker (2015). "Regret theory: a bold alternative to the alternatives.” Economic Journal 125: 493-532.

Bolton, Gary, Axel Ockenfels, and Julia Stauf (2015). "Social responsibility promotes conservative risk behavior.” European Economic Review 74: 109-127.

Cadsby, Charles Bram, and Elizabeth Maynes (1999). "Voluntary provision of threshold public goods with continuous contributions: experimental evidence." Journal of Public Economics 71 (1): 53-73.

Charness, Gary, and Matthew O. Jackson (2009). "The role of responsibility in strategic risk-taking." Journal of Economic Behavior \& Organization 69: 241-247.

Charness, Gary, Luca Rigotti, and Aldo Rustichini (2007). "Individual behavior and group membership.” American Economic Review 97 (4): 1340-1352.

Croson, Rachel, and Melanie Marks (2000). "Step returns in threshold public goods: a meta-analysis and experimental analysis.” Experimental Economics 2 (3): 239-259.

Dannenberg, Astrid, Andreas Löschel, Gabriele Paolacci, Christiane Reif, and Alessandro Tavoni (2015). On the provision of public goods with probabilistic and ambiguous thresholds. Environmental and Resource Economics 61: 365-383.

Habla, Wolfgang and Ralph Winkler. "Strategic Delegation and International Permit Markets: Why Linking May Fail.” EfD Discussion Paper Series 16-12 (2016).

Hamman, John R., Roberto A. Weber, and Jonathan Woon (2011). "An experimental investigation of electoral delegation and the provision of public goods." American Journal of Political Science 55 (4): 738-752.

İriş, Doruk, Jungmin Lee and Alessandro Tavoni. "Delegation and public pressure in a threshold public goods game: theory and experimental evidence". Grantham Research Institute working paper 186 (2015).

İriş, Doruk (2018). "Representation and social regret-aversion in risk-taking." The Korean Journal of Industrial Organization 26 (3): 1-17. 
Kocher, Martin G., Fangfang Tan, and Jing Yu (2018). Providing Global Public Goods: Electoral Delegation and Cooperation. Economic Inquiry, Vol. 56 (1): 381-398.

Loomes, Graham, and Robert Sugden (1982). "Regret Theory: An alternative theory of rational choice under uncertainty." The Economic Journal 92 (368): 805-824.

Milinski, Manfred, Ralf D. Sommerfeld, Hans-Jürgen Krambeck, Floyd A. Reed, and Jochem Marotzke (2008). "The collective-risk social dilemma and the prevention of simulated dangerous climate change." Proceedings of the National Academy of Sciences USA 105 (7): 2291-2294

Song, Fei (2008). "Trust and reciprocity behavior and behavioral forecasts: Individual versus group-representatives." Games and Economic Behavior 62: 675-696.

Stoop, Jan, Charles N. Noussair, and Daan van Soest (2012). "From the lab to the field: cooperation among fishermen." Journal of Political Economics 120 (6): 1027-1056.

Tavoni, Alessandro, Astrid Dannenberg, Giorgos Kallis, and Andreas Löschel (2011). "Inequality, communication and the avoidance of disastrous climate change in a public goods game." Proceedings of the National Academy of Sciences USA 108: $11825-11829$.

Zeelenberg, Marcel (1999). “Anticipated regret, expected feedback and behavioral decision making." Journal of Behavioral Decision Making 12 (1): 93-106. 


\section{Figure 1: Hierarchical Decision-making in Delegation Treatments}

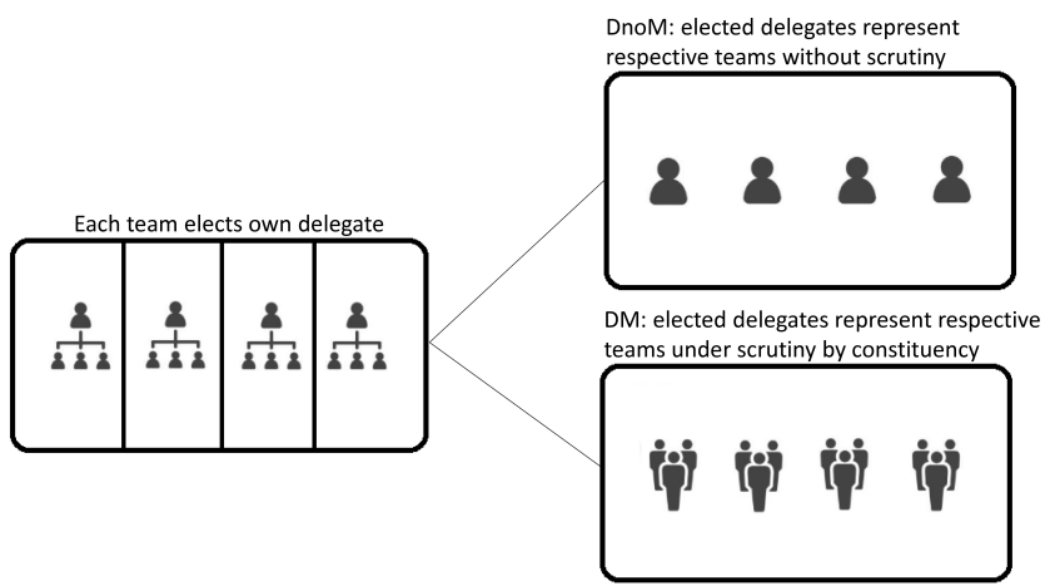

Figure 2. Average Group Contributions across Treatments

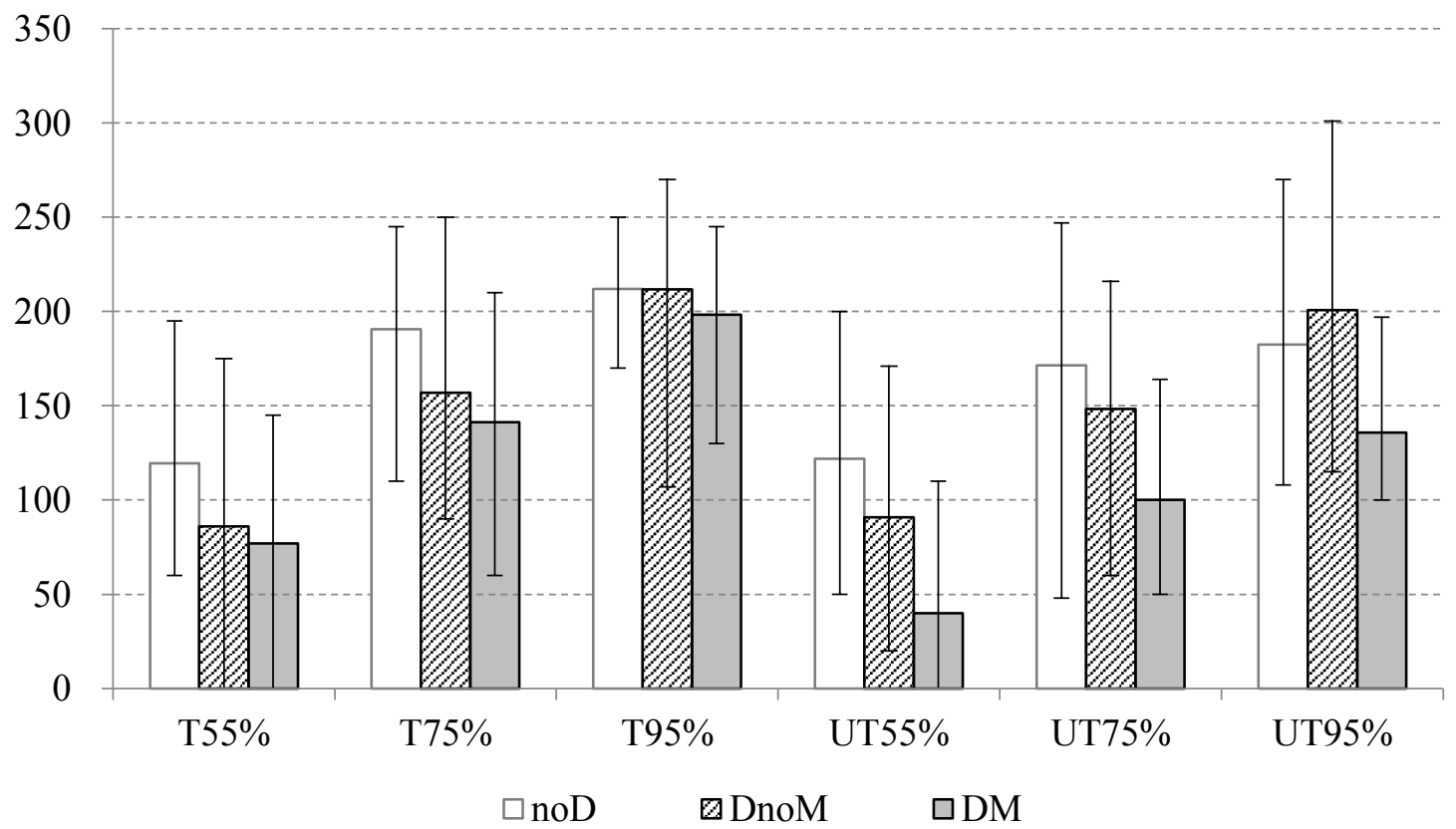

Notes: $\mathrm{T} 55 \%=$ Certain threshold with probability of loss $55 \%$; T75\% $=$ Certain threshold with probability of loss $75 \%$; T95\% = Certain threshold with probability of loss 95\%; UT55\%= Uncertain threshold with probability of loss 55\%; UT75\% = Uncertain threshold with probability of loss 75\%; UT95\% = Uncertain threshold with probability of loss $95 \%$. Full contribution is $480(120 * 4)$. The bars indicate minimum-maximum contributions among 8 groups in each treatment. 
Table 1: Main Parameters and Equilibria

\begin{tabular}{|c|c|c|c|c|c|c|c|}
\hline & \multirow{2}{*}{$\begin{array}{c}\text { Practice } \\
\text { Phase }\end{array}$} & \multicolumn{7}{|c|}{ Game Phase } \\
\cline { 3 - 8 } & Round 1 & Round 2 & Round 3 & Round 4 & Round 5 & Round 6 \\
\hline $\begin{array}{c}\text { Loss } \\
\text { Uncertainty } \\
(\boldsymbol{p})\end{array}$ & 0.55 & 0.75 & 0.55 & 0.95 & 0.95 & 0.55 & 0.75 \\
\hline $\begin{array}{c}\text { Threshold } \\
\text { Location } \\
\text { Uncertainty }\end{array}$ & $\begin{array}{c}\text { Uncertain } \\
T\left(T_{1}=190\right. \\
\left.T_{2}=290\right)\end{array}$ & $\begin{array}{c}\text { Certain } T \\
(T=240)\end{array}$ & $\begin{array}{c}\text { Certain } T \\
(T=240)\end{array}$ & $\begin{array}{c}\text { Certain } T \\
(T=240)\end{array}$ & $\begin{array}{c}\text { Uncertain } \\
T\left(T_{1}=190\right. \\
\left.T_{2}=290\right)\end{array}$ & $\begin{array}{c}\text { Uncertain } \\
T\left(T_{1}=190\right. \\
\left.T_{2}=290\right)\end{array}$ & $\begin{array}{c}\text { Uncertain } \\
T\left(T_{1}=190\right. \\
\left.T_{2}=290\right)\end{array}$ \\
\hline $\begin{array}{c}\text { Damage Rate } \\
(1-q)\end{array}$ & 0.9 & 0.9 & 0.9 & 0.9 & 0.9 & 0.9 & 0.9 \\
\hline $\begin{array}{c}\text { Highest } \\
\text { Expected } \\
\text { Payoff } \\
\text { Equilibria }\end{array}$ & $\mathrm{NC}$ & $\mathrm{SPC}$ & $\mathrm{NC}=\mathrm{SPC}$ & $\mathrm{SPC}$ & $\mathrm{SPC} 2$ & $\mathrm{NC}$ & $\mathrm{SPC} 1$ \\
\hline
\end{tabular}

Table 2. Treatment Effects on Group Contribution

\begin{tabular}{lccccc}
\hline & $(1)$ & $(2)$ & $(3)$ & $(4)$ & $(5)$ \\
& All & $\mathrm{p}=55 \%$ & $\mathrm{p}=75 \%$ & $\mathrm{p}=95 \%$ & First round \\
\hline & & & & & \\
DnoM & -17.146 & -32.188 & -28.313 & 9.062 & -33.625 \\
& $(18.958)$ & $(25.156)$ & $(24.151)$ & $(21.029)$ & $(25.116)$ \\
DM & $-50.917^{* * *}$ & $-62.188^{* *}$ & $-60.375^{* * *}$ & -30.188 & $-49.375^{*}$ \\
& $(17.906)$ & $(23.646)$ & $(21.483)$ & $(17.948)$ & $(25.463)$ \\
Loss prob. $=75 \%$ & $62.271^{* * *}$ & & & & \\
& $(8.856)$ & & & & \\
Loss prob. $=95 \%$ & $100.979^{* * *}$ & & & & \\
& $(10.191)$ & & & & \\
Uncertain thresholds & $-22.417^{* *}$ & -9.833 & $-23.042^{*}$ & $-34.375^{* *}$ & \\
& $(8.652)$ & $(9.025)$ & $(11.806)$ & $(13.170)$ & \\
Constant & $123.062^{* * *}$ & $125.542^{* * *}$ & $192.521^{* * *}$ & $214.375^{* * *}$ & $190.625^{* * *}$ \\
& $(14.041)$ & $(18.663)$ & $(16.831)$ & $(12.450)$ & $(16.241)$ \\
& & & & & \\
\hline Observations & 144 & 48 & 48 & 48 & 0.152 \\
R-squared & 0.471 & 0.209 & 0.225 & 0.193 & \\
\hline
\end{tabular}

Notes: OLS Estimation. Robust standard errors, clustered by group, are presented in parentheses. Each group played 6 rounds. Significance level: *** $1 \%, * * 5 \%, * 10 \%$. 
Table 3. Determinants of Team Contribution: Individual Fixed Effect

\begin{tabular}{|c|c|c|c|c|c|c|c|c|}
\hline \multirow[t]{2}{*}{ Treatment } & \multirow{2}{*}{$\begin{array}{c}\text { noD } \\
(1)\end{array}$} & \multicolumn{3}{|c|}{ DnoM } & \multicolumn{4}{|c|}{$\mathrm{DM}$} \\
\hline & & (2) & (3) & (4) & $(5)$ & (6) & (7) & $(8)$ \\
\hline Avg. pledge by others & $\begin{array}{l}-0.040 \\
(0.102)\end{array}$ & $\begin{array}{c}0.053 \\
(0.066)\end{array}$ & $\begin{array}{c}0.053 \\
(0.066)\end{array}$ & $\begin{array}{c}0.051 \\
(0.067)\end{array}$ & $\begin{array}{c}0.039 \\
(0.047)\end{array}$ & $\begin{array}{c}0.061 \\
(0.051)\end{array}$ & $\begin{array}{c}0.065 \\
(0.049)\end{array}$ & $\begin{array}{c}0.060 \\
(0.137)\end{array}$ \\
\hline Avg. expectation by others & $\begin{array}{l}-0.044 \\
(0.213)\end{array}$ & $\begin{array}{c}0.319 \\
(0.235)\end{array}$ & $\begin{array}{c}0.319 \\
(0.237)\end{array}$ & $\begin{array}{c}0.325 \\
(0.231)\end{array}$ & $\begin{array}{c}-0.186 \\
(0.137)\end{array}$ & $\begin{array}{l}-0.169 \\
(0.166)\end{array}$ & $\begin{array}{c}-0.183 \\
(0.163)\end{array}$ & $\begin{array}{c}0.187 \\
(0.328)\end{array}$ \\
\hline $75 \%$ chance of loss & $\begin{array}{c}18.739 * * * \\
(5.353)\end{array}$ & $\begin{array}{c}15.068^{* *} \\
(5.541)\end{array}$ & $\begin{array}{c}15.082 * * * \\
(5.440)\end{array}$ & $\begin{array}{c}15.154 * * * \\
(5.487)\end{array}$ & $\begin{array}{c}15.084 * * * \\
(5.336)\end{array}$ & $\begin{array}{l}8.177 * \\
(4.733)\end{array}$ & $\begin{array}{l}8.733 * \\
(4.734)\end{array}$ & $\begin{array}{l}13.595 \\
(8.665)\end{array}$ \\
\hline $95 \%$ chance of loss & $\begin{array}{c}24.859 * * * \\
(5.243)\end{array}$ & $\begin{array}{c}30.286^{* * *} \\
(6.528)\end{array}$ & $\begin{array}{c}30.303 * * * \\
(6.562)\end{array}$ & $\begin{array}{c}30.554 * * * \\
(6.623)\end{array}$ & $\begin{array}{c}29.065^{* * *} * \\
(6.266)\end{array}$ & $\begin{array}{c}16.877 * * * \\
(5.758)\end{array}$ & $\begin{array}{c}16.889^{* * *} \\
(5.921)\end{array}$ & $\begin{array}{c}19.470 * * \\
(9.073)\end{array}$ \\
\hline Uncertain thresholds & $\begin{array}{l}-3.878 \\
(5.494)\end{array}$ & $\begin{array}{l}-4.512 \\
(5.928)\end{array}$ & $\begin{array}{l}-4.508 \\
(5.961)\end{array}$ & $\begin{array}{l}-4.216 \\
(5.954)\end{array}$ & $\begin{array}{c}-9.601^{*} \\
(5.333)\end{array}$ & $\begin{array}{c}-12.065 * * \\
(4.761)\end{array}$ & $\begin{array}{c}-11.495 * * \\
(4.729)\end{array}$ & $\begin{array}{c}-21.257^{* *} \\
(9.235)\end{array}$ \\
\hline \multicolumn{9}{|l|}{ Team members' opinions } \\
\hline Average & & & $\begin{array}{c}-0.001 \\
(0.116)\end{array}$ & & & $\begin{array}{c}0.483 * * * \\
(0.119)\end{array}$ & & \\
\hline Lower & & & & $\begin{array}{c}0.025 \\
(0.089)\end{array}$ & & & $\begin{array}{c}0.342 * * * \\
(0.115)\end{array}$ & $\begin{array}{l}0.411 * \\
(0.213)\end{array}$ \\
\hline Higher & & & & $\begin{array}{c}-0.032 \\
(0.107)\end{array}$ & & & $\begin{array}{c}0.136 \\
(0.084)\end{array}$ & $\begin{array}{l}-0.334 \\
(0.298)\end{array}$ \\
\hline Constant & $\begin{array}{l}38.342 * \\
(22.410)\end{array}$ & $\begin{array}{c}-4.724 \\
(12.597)\end{array}$ & $\begin{array}{c}-4.705 \\
(12.692)\end{array}$ & $\begin{array}{c}-3.931 \\
(13.892)\end{array}$ & $\begin{array}{l}-15.904 \\
(18.516)\end{array}$ & $\begin{array}{l}-13.386 \\
(19.755)\end{array}$ & $\begin{array}{l}-12.074 \\
(19.091)\end{array}$ & $\begin{array}{c}19.275 \\
(32.907)\end{array}$ \\
\hline Team FE & $\mathrm{Y}$ & $\mathrm{Y}$ & $\mathrm{Y}$ & $\mathrm{Y}$ & $\mathrm{Y}$ & $\mathrm{Y}$ & $\mathrm{Y}$ & $\mathrm{Y}$ \\
\hline Round FE & $\mathrm{Y}$ & $\mathrm{Y}$ & $\mathrm{Y}$ & $\mathrm{Y}$ & $\mathrm{Y}$ & $\mathrm{Y}$ & $\mathrm{Y}$ & $\mathrm{Y}$ \\
\hline Observations & 192 & 192 & 192 & 192 & 192 & 192 & 192 & 99 \\
\hline R-squared & 0.478 & 0.588 & 0.588 & 0.589 & 0.614 & 0.672 & 0.676 & 0.604 \\
\hline
\end{tabular}

from the data (93 cases). Significance level: $* * * 1 \%, * * 5 \%, * 10 \%$. 
Table 4. Average Contributions of Delegates and Non-delegates

\begin{tabular}{|c|c|c|c|c|c|c|c|}
\hline & \multicolumn{3}{|c|}{ DnoM } & \multicolumn{3}{|c|}{$\mathrm{DM}$} & \multirow[b]{2}{*}{$\begin{array}{c}\text { Non- } \\
\text { delegates } \\
\text { DnoM = } \\
\text { DM }\end{array}$} \\
\hline & $\begin{array}{c}\text { Non- } \\
\text { delegates }\end{array}$ & Delegates & $\begin{array}{c}\text { Non- } \\
\text { delegates = } \\
\text { Delegates }\end{array}$ & $\begin{array}{c}\text { Non- } \\
\text { delegates }\end{array}$ & Delegates & $\begin{array}{c}\begin{array}{c}\text { Non- } \\
\text { delegates } \\
=\end{array} \\
\text { Delegates }\end{array}$ & \\
\hline \multirow{2}{*}{$\mathrm{T} 55 \%$} & 27.94 & 21.50 & 6.44 & 25.47 & 19.22 & 6.25 & 2.47 \\
\hline & $(25.81)$ & $(25.17)$ & {$[0.25]$} & (26.97) & $(25.12)$ & {$[0.28]$} & [0.74] \\
\hline \multirow[t]{2}{*}{$\mathrm{T} 75 \%$} & 40.45 & 39.25 & 1.20 & 38.95 & 35.31 & 3.64 & 1.50 \\
\hline & (25.58) & $(25.22)$ & {$[0.83]$} & (26.28) & (26.94) & {$[0.53]$} & {$[0.60]$} \\
\hline \multirow[t]{2}{*}{ Т95\% } & 42.97 & 52.94 & -9.97 & 49.30 & 49.56 & -0.26 & -6.33 \\
\hline & (31.67) & (20.08) & {$[0.11]$} & (24.05) & (23.62) & [0.96] & {$[0.21]$} \\
\hline \multirow[t]{2}{*}{ UT55\% } & 26.02 & 22.72 & 3.30 & 26.61 & 10.00 & 16.61 & -0.59 \\
\hline & (25.69) & (28.38) & {$[0.57]$} & (28.90) & (20.32) & {$[0.00]$} & [1.00] \\
\hline \multirow[t]{2}{*}{ UT75\% } & 45.05 & 37.09 & 7.96 & 44.03 & 25.00 & 19.03 & 1.02 \\
\hline & (30.18) & $(28.82)$ & {$[0.22]$} & (30.08) & $(28.30)$ & {$[0.00]$} & {$[0.90]$} \\
\hline \multirow[t]{2}{*}{ UT95\% } & 52.97 & 50.19 & 2.78 & 52.95 & 33.94 & 19.01 & 0.02 \\
\hline & $(30.01)$ & $(25.12)$ & {$[0.65]$} & (27.69) & $(30.11)$ & {$[0.00]$} & {$[0.85]$} \\
\hline
\end{tabular}

Notes: Standard deviations are presented in parentheses. P-values of equality tests are presented in the last column. 